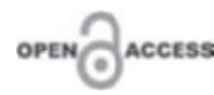

\title{
Baixa Dose de Prednisona de Liberação Modificada em Espondiloartrite Axial: Eficácia e Tolerabilidade de Três Meses
}

\author{
Low Dose of Prednisone of Modified Release in Aponal \\ Spondylarthritis: Efficacy and Tolerability of Three Months
}

Francesca Bandinelli ${ }^{1}$, Francesco Scazzariello ${ }^{1}$,Emanuela Pimenta da Fonseca ${ }^{2}$, Mittermayer Barreto Santiago $^{2}$, Claudio Marcassa ${ }^{3}$, Francesca Nacci $^{1}$, Marco Matucci Cerinic ${ }^{1}$

${ }^{1}$ Departamento de Medicina Experimental e Clínica, Universidade de Florença; Florença, Itália; ${ }^{2}$ Serviço de Reumatologia do Hospital Santa Izabel, Escola Bahiana de Medicina e Saúde Pública; Salvador, Bahia, Brasil; ${ }^{3}$ Maugeri Institutos Clínicos e Cientificos; Veruno, Itália

Correspondence addresses: Dr. Mittermayer Santiago mitter@svn.com.br

Received: October 24, 2018

Revised: December 17, 2018

Accepted: January 13, 2019

Published: March 27, 2019

Data Availability Statement: All relevant data are within the paper and its Supporting Information files.

Competing interests: The authors have declared that no competing interests exist.

\section{Copyright}

(C) 2019 by Santa Casa de Misericórdia da Bahia.

All rights reserved.

ISSN: 2526-5563
Os glicocorticoides orais (GCs) demonstraram ser eficazes na redução dos sintomas inflamatórios da artrite reumatoide, mas seu uso não é apoiado por evidências científicas nas espondiloartrites (SpA). A prednisona oral de liberação modificada (LM) feita na hora de dormir tem se mostrado mais eficaz do que a prednisona de liberação imediata tomada pela manhã. Este estudo de coorte unicêntrico avaliou, retrospectivamente, a eficácia e a segurança de prednisona de LM em baixas doses, por 12 semanas ( $5 \mathrm{mg}$ ao dia, administração ao deitar) em 57 pacientes adultos com SpA axial. Destes, 41 apresentavam doença ativa (BASDAI $\geq 4)$ no início do estudo. Uma melhoria de $50 \%$ do BASDAI ou um BASDAI menor que 4 no final do estudo foi o desfecho primário. A prednisona de LM reduziu significativamente o BASDAI (de $5,5 \pm 2,6$ para $3,0 \pm 2,8 ; p<0,001$ ), assim como sintomas inflamatórios, dor, fadiga e rigidez matinal. No total, sete pacientes $(11,8 \%)$ tiveram reações adversas não graves após a prednisona de LM. Em pacientes com SpA sintomática, o uso de baixa dose de prednisonade LM reduziu os sintomas e os índices clínicos de atividade da doença, além de mostrar um perfil de segurança positivo.

Palavras-chave: Espondiloartrite; Glicocorticoides; Prednisona modificada.

Oral glucocorticoids (GCs) have been shown to be effective in reducing the inflammatory symptoms of rheumatoid arthritis, but their use is not supported by scientific evidence on spondyloarthritis (SpA). Modified release oral prednisone (LM) at bedtime has been shown to be more effective than immediate-release prednisone taken in the morning. This unicentric cohort study retrospectively evaluated the efficacy and safety of low dose LM prednisone for 12 weeks ( $5 \mathrm{mg}$ daily, bedtime administration) in 57 adult patients with axial SpA. Forty-one of these patients showed active disease (BASDAI $\geq 4$ ) at baseline. A 50\% improvement in BASDAI or a BASDAI less than 4 at the end of the study was the primary endpoint. LM prednisone significantly reduced BASDAI (from $5.5 \pm 2.6$ to $3.0 \pm 2.8, \mathrm{p}<0.001$ ), as well as inflammatory symptoms, pain, fatigue and morning stiffness. In total, seven patients $(11.8 \%)$ had non-serious adverse reactions after LM prednisone. In patients with symptomatic SpA, the use of low dose LM prednisolone reduced the symptoms and clinical ovidences of disease activity, in addition to showing a positive safety profile.

Keywords: Spondylarthritis; Glucocorticoids; Modified prednisone. 


\section{Introdução}

O objetivo do tratamento da espondiloartrite (SpA) é o controle dos sinais e sintomas e a redução dos parâmetros da inflamação. Os anti-inflamatórios não esteroides (AINEs) são recomendados como tratamento farmacológico de primeira linha; e para aqueles pacientes que não respondem aos AINHs, são utilizados os inibidores do TNF- $\alpha$.

Ao contrário da artrite reumatoide (AR), nem as drogas antirreumáticas convencionais modificadoras da doença (DMARDs) nem os glicocorticoides (GCs) são recomendados no tratamento da $\mathrm{SpA}$ axial, ${ }^{1}$ e a evidência de sua eficácia neste contexto é limitada e controversa. No entanto, os GCs podem ser úteis em pacientes com envolvimento periférico, particularmente em pacientes intolerantes ou com contraindicações aos AINEs.

A eficácia da dose baixa de prednisona em pacientes com SpA é desconhecida. Assim, o objetivo deste estudo preliminar foi avaliar a eficácia clínica a médio prazo e o perfil de segurança de baixas doses de prednisona de liberação modificada (LM), ou seja, liberação do medicamento no início da manhã em pacientes com SpA refratária ou intolerante a AINES ou com comorbidades que limitam o uso dos agentes bloqueadores do TNF- $\alpha$.

\section{Material e Métodos}

Foi realizada uma análise retrospectiva observacional de 12 semanas, em um único centro usando dados de pacientes tratados na Unidade Reumatológica da Universidade de Florença, Itália. Todos os pacientes assinaram o consentimento informado para a coleta anônima de seus dados demográficos e clínicos.

Foram revisados os dados de todos os pacientes adultos virgens de tratamento com GCs com idade acima 18 anos, com diagnóstico de SpA axial de acordo com os critérios da $\mathrm{ASAS}^{2}$, de setembro de 2012 a dezembro de 2013. Foram incluídos neste estudo aqueles pacientes refratários ou intolerantes aos AINES e/ou com comorbidades que limitavam o uso dos agentes bloqueadores do TNF- $\alpha$ e que foram tratados com prednisona de LM.
Os pacientes foram avaliados por reumatologistas com experiência no tratamento de $\mathrm{SpA}$ e receberam $5 \mathrm{mg} /$ dia de prednisona de LM (Lodotra ${ }^{\circledR}$, administração antes de dormir, às $22 \mathrm{~h} 00$ ). Os pacientes foram atendidos ou contatados por telefone ou e-mail após 4 semanas; se bem tolerado, nenhum ajuste de dose de prednisona de LM foi feito; caso contrário, foi interrompido. Todos os pacientes foram reavaliados 8 semanas depois e, assim, após 12 semanas em uso de prednisona de LM.

Parâmetros de eficácia e segurança

A atividade da doença foi medida no início do estudo e na semana 12 pelo BASDAI, de acordo com a recomendação EULAR ${ }^{3}$. Fadiga, duração da rigidez matinal (em minutos) assim como a mobilidade espinhal, medida pelo Índice de Metrologia de Espondilite Anquilosante de Bath ${ }^{4}$ foram também avaliados. Outras avaliações incluíram a entesite, usando o Escore de Entesite para Espondilite Anquilosante de Maastricht (MASES de 0-13); ${ }^{5}$ Avaliações de resultados laboratoriais incluíram velocidade de sedimentação de hemácias (VHS) e níveis de proteína $C$ reativa (PCR).

Avaliações de segurança também foram realizadas com o registro de reações adversas relacionadas à droga que ocorreram após a primeira ingestão da prednisona de LM.

\section{Resultados}

No total, 93 pacientes foram selecionados e 59 preencheram os critérios de seleção. Dois deles pararam a prednisona de LM precocemente por razões não clínicas (má adesão) e foram excluídos; os 57 restantes foram incluídos na análise final. Todos completaram o follow-up de 12 semanas. A idade mediana dos pacientes foi 56 anos, faixa de 26-86 anos; $65 \%$ mulheres. No início do estudo, três quartos dos pacientes $(72,9 \%)$ foram tratados com drogas DMARDs e/ou anti-TNF- $\alpha$.

Após 12 semanas com prednisona de LM $(5 \mathrm{mg} /$ dia), observou-se uma redução significativa do BASDAI na população geral. Ador axial e periférica diminuiu de $5,2 \pm 3,9$ para $3,4 \pm 3,5(\mathrm{p}=0,0013)$ e de $6,3 \pm 3,7$ para $3,8 \pm 3,7(\mathrm{p}=0,001)$, respectivamente. 
A fadiga e a rigidez matinal também diminuíram significativamente. Ao contrário, apenas modestas variações no BASMI e no MASES foram registradas (de $1,5 \pm 1,9$ para $1,2 \pm 1,6$ e de $2,9 \pm 4,3$ para $1,9 \pm 3,7$, respectivamente; não significante para ambas as variações).

Os marcadores inflamatórios também diminuíram significativamente após a prednisona de LM: a VHS diminuiu de $20,3 \pm 16$ para $15,4 \pm 13 \mathrm{~mm}(\mathrm{p}<0,001)$ e PCR de $11 \pm 26$ para $4 \pm 6 \mathrm{mg} / \mathrm{L}(\mathrm{p}<0,05)$.

No geral, a taxa de resposta após 12 semanas foi de 52,6\%: entre os 16 pacientes que iniciaram a prednisona de LM com uma doença pouco ativa (pontuação BASDAI $<4$ ), o desfecho primário (ou seja, uma melhoria de $50 \%$ no BASDAI na semana 12) foi alcançado em oito (50\%). Uma taxa de resposta semelhante ( $\mathrm{n}=22,53,7 \%$, não significativo) foi documentada nos 41 pacientes com SpA ativa no início do estudo.

Quanto à segurança, o novo tratamento foi bem tolerado durante o período de 12 semanas de observação sendo relatados apenas eventos adversos moderados em seis pacientes e um evento adverso grave em uma paciente.

\section{Discussão}

Embora os esteroides administrados localmente tenham se mostrado eficazes quando usados por injeções guiadas por tomografia computadorizada nas articulações sacroilíacas, as recomendações ASAS / EULAR para o manejo de SpA ainda afirmam que o uso de GCs sistêmicos não é apoiado por evidências. ${ }^{4}$ No presente estudo, a eficácia da prednisona de LM foi avaliada em condições reais durante um período de observação de 3 meses. Apesar das baixas dosagens de esteroides prescritas, os autores encontraram uma melhora substancial de fadiga, dor e rigidez matinal, uma redução significativa nos marcadores inflamatórios, bem como nos escores do BASDAI. Pelo contrário, a dor sacroilíaca e o escore BASMI permaneceram inalterados após 12 semanas, e o MASES diminuiu apenas ligeiramente após a prednisona de LM. A dor sacroilíaca é um parâmetro de eficácia difícil de ser avaliado e raramente usado para monitorar as melhorias da SpA em ensaios clínicos. O BASMI, um parâmetro que indica perda de mobilidade axial, e indiretamente, danos permanentes, não surpreendentemente, foi maior em pacientes com baixa atividade da doença, que também apresentavam maior duração da doença.

O estudo também demonstrou que $42(53,7 \%)$ dos pacientes com SpA ativa no início do estudo respondeu ao tratamento, apesar das doses muito baixas (5 $\mathrm{mg}$ por dia) de prednisona de LM prescrita, com uma redução no escore BASDAI de -3,1 pontos após 3 meses. Além disso, a dor e a fadiga axial e periférica e a rigidez matinal já melhoraram significativamente após 4 semanas e depois permaneceram substancialmente inalteradas.

No geral, os perfis de segurança e tolerabilidade do tratamento com doses baixas de prednisona por LM foram bons neste período de 12 semanas de estudo.

Este estudo apresenta várias limitações, incluindo o desenho retrospectivo, a ausência de um grupo controle e o curto período de observação. No entanto, a população estudada é bem representativa de pacientes tipicamente encontrados na prática dos reumatologistas.

\section{Referências}

1. Braun J, van den Berg R, Baraliakos X, et al. 2010 updateofthe ASAS/EULAR recommendations for the management of ankylosing spondylitis. Ann Rheum Dis. 2011;70(6):896-904.

2. Rudwaleit M, van der Heijde D, Landewe R, et al. The development of assessment of spondyloarthritis international society classification criteria for axial spondyloarthritis (part II): validation and final selection. Ann Rheum Dis. 2009;68(6):777-83.

3. Smolen JS, Landewe R, Breedveld FC, et al. EULAR recommendations for the management of rheumatoidarthritis with synthetic and biological disease-modifying antirheumatic drugs. Ann Rheum Dis. 2010;69(6):964-75.

4. Jones SD, Porter J, Garrett SL, Kennedy LG, Whitelock $\mathrm{H}$, Calin A. A new scoring system for the Bath Ankylosing Spondylitis Metrology Index (BASMI). J Rheumatol. 1995;22(8):1609.

5. Heuft-DorenboschL, Spoorenberg A, vanTubergen A, et al. Assessment of enthesitis in ankylosing spondylitis. Ann Rheum Dis. 2003;62(2): 127-32. 